\title{
Revitalización del espacio público sobre una línea férrea subutilizada en el cantón Durán, Guayaquil
}

Richard Ronquillo Salas

rronquillos@ulvr.edu.ec

Silvia Herrera Ferrín

Sherreraf@ulvr.edu.ec

\section{Carolina Morales Robalino}

lmoralesr@ulvr.edu.ec

Universidad Laica Vicente Rocafuerte de Guayaquil

Guayaquil - Ecuador

\section{RESUMEN}

Este trabajo se enfoca en presentar un análisis y una propuesta sobre un espacio público que se encuentra interceptado por una línea férrea en estado de abandono, situada en la parroquia El Recreo del cantón Durán, provincia del Guayas. Con el fin de obtener la información necesaria para poder realizar el diagnostico de las variables urbanas, según la metodología del urbanista Jan Ghel, se consideraron los siguientes 5 ámbitos de actuación: la preservación del patrimonio, movilidad sustentable, equidad y diversidad, escala humana y el aspecto económico y cultural. Como resultado del trabajo, se logra obtener datos cuantitativos y cualitativos de las necesidades presentadas por los moradores del sector, siendo una de estas el tema de la inseguridad, por el cual más del $50 \%$ expresa del sitio como un lugar inseguro, y un 70,11\% afirma que necesitan que el sector tenga una revitalización, con el objetivo de promover la circulación peatonal segura, brindar espacios socioculturales, implementar espacios que mejoren la experiencia visual y fomentar una relación entre la vida pública y el desarrollo económico, con el fin de revitalizar y mejorar la calidad de vida de los residentes del sector analizado.

Palabras clave: revitalizar; diagnostico; desarrollo; metodología; sustentabilidad 


\title{
Revitalization of public space on an underutilized railway line in the Durán canton, Guayaquil
}

\begin{abstract}
This work focuses on presenting an analysis and a proposal on a public space that is intercepted by a railway line in a state of abandonment, located in the El Recreo parish of the Duran canton, Guayas province. Know the characteristics that the sector lacks and the strengths it currently possesses, in such a way that it is possible to identify the weaknesses and opportunities of the urban space. In order to obtain the necessary information to be able to carry out the diagnosis of urban variables according to the methodology of the urban planner Jan Gehl. For which the following 5 areas of action were considered, which are: heritage preservation, sustainable mobility, equity and diversity, human scale, and the economic and cultural aspect. As a result of the work, it is possible to defend the historical value of the site, promote greater importance for pedestrian circulation, provide socio-cultural spaces, implement spaces that improve the visual experience and foster a relationship between public life and economic development, in order to revitalize and improve the quality of life of the residents of the analyzed sector.
\end{abstract}

Keywords: revitalize; diagnosis; developing; methodology; sustainability

Artículo recibido: 15 enero 2022 Aceptado para publicación: 08 febrero 2022 Correspondencia: rronquillos@ulvr.edu.ec Conflictos de Interés: Ninguna que declarar 


\section{INTRODUCCIÓN}

El cantón Durán es uno de los polos industriales del estado ecuatoriano y el principal punto de conexión de transporte multimodal, pero con una inapropiada planificación que por medio de sus puertos, carreteras y ferrocarril, formando un desorden estructural que se ha ido desarrollando década tras década, se convierte en un desafío aprovechar el potencial de la ciudad para el alcance de nuevas oportunidades de vida de la población, ya que el crecimiento económico debe estar relacionado con el "Buen Vivir", es decir “proyectar desde y para la gente, logrando generar espacios democráticos y multisociales, que tanto sirva para la población local como también para los visitantes”. (Arrieta, 2017). Siendo así el motivo por el cual, la estructura urbana de la ciudad se deberá planificar y organizar en diferentes zonas, por ende, se plantea el estudio al tema referido como la revitalización urbana del espacio público en el sector de la línea férrea en la 5ta y 3era etapa de la ciudadela el Recreo, basado en la necesidad de rehabilitar la peatonalización del tramo lineal para el beneficio de los habitantes. Constantemente se observa la desigualdad y el desequilibrio en la provisión de áreas verdes para una buena calidad de vida "cuyo diseño intenta mejorar la calidad ambiental, la oportunidad ambiental, económica o el valor estético asociado con el paisaje urbano" (Sorensen Mark, 1998) ; además de la inadecuada concepción y el valor que se les da a muchos sitios abiertos de uso público debido a que estos espacios se tornan desperdiciados principalmente por el desorganizado emplazamiento del territorio a medida que aumenta el esparcimiento social en el transcurso del tiempo.

Por tal motivo, el Plan de Gobierno Municipal establece que se requiere un modelo de desarrollo sostenible y socialmente incluyente en donde manifiesta dos frentes, el primero es el reordenamiento del entramado industrial y el segundo es la revitalización de los espacios públicos mediante la integración de la cultura e historia del cantón, teniendo la vía del ferrocarril como uno de los pilares estructurantes sobre el cual se necesita recuperar las zonas degradadas para un desarrollo urbanístico sostenible, es decir un crecimiento articulado y armónico "que constituye el progreso de un lugar y/o territorio específico, regulando el avance del hombre con su entorno y estableciendo una relación armoniosa entre lo económico, lo social, lo ambiental, lo cultural y/o el sistema de valores". (Plinio, 2018) 
El Plan Estratégico Institucional manifiesta que la simbiosis entre el sector privado y público en beneficio de las personas es uno de los hechos que la Municipalidad del cantón desea alcanzar, ya que la relación de estos puede conllevar varios beneficios que efectúe un cambio significativo de la urbe. Por ende, la iniciativa de considerar áreas vacantes para el fomento de una vida sustentable de forma activa se orienta a las expectativas que contiene la política general del Municipio como "Promover y contribuir al Buen Vivir de los ciudadanos, a través del impulso del desarrollo económico sostenible, solidario y ambientalmente responsable”. (GAD Durán 2019)

Por consiguiente, el interés sobre el estado de estos espacios vacantes disminuye generalmente ya que no todas las personas tienen un fácil acceso por diferentes motivos como la delincuencia, las dificultades de movilidad y accesibilidad por la existencia de barreras físicas, dificultades económicas para una rehabilitación y el descuido por parte de los ciudadanos y entidades privadas o estatales. Por lo tanto, el tramo lineal en donde se lleva a cabo el trayecto del Ferrocarril cantonal, que conecta dos etapas de la ciudadela El Recreo, existe un crecimiento de concentración social que tiene la necesidad de transitar en este sector para llevar a cabo sus actividades de movilización que en efecto ocasiona una involuntaria apropiación de espacios para la recreación al aire libre pero inadecuado por su condición; que según el Proyecto de Ley Orgánica de Ordenamiento Territorial, Uso y Gestión de Suelo en el art 415 detalla: "El Estado central y los gobiernos autónomos descentralizados adoptarán políticas integrales y participativas de ordenamiento territorial urbano y de uso del suelo, que permitan regular el crecimiento urbano, el manejo de la fauna urbana e incentiven el establecimiento de zonas verdes".

De acuerdo a los objetivos institucionales del Gobierno Autónomo Descentralizado de Durán, se establece en el ítem 2.2 del sistema de sostenibilidad ambiental que se debe "Recuperar las zonas naturales del cantón, para contar con espacios que permitan reducir y compensar la huella de carbono que genera la industria local y como recurso para el desarrollo de actividades familiares y turísticas con énfasis en inclusión social”. Por lo tanto, el restablecimiento del lugar contribuye al sector como el inicio de un destino armónico en donde coexista la industria responsable con el aprovechamiento de las riquezas naturales que fortalezcan el desarrollo turístico-ecológico. 


\section{Medidas de Bioseguridad ante el Covid-19}

Debido a la gravedad y longevidad del evento pandémico por el Covid-19 la OMS lo declaró el 11 de marzo del 2020 como emergencia global, mientras que en Ecuador con el acuerdo ministerial N. ${ }^{\circ}$ 00126-2020 emitido por la Ministra de Salud, declara Estado de emergencia sanitaria al Sistema Nacional de la Salud (GADMCD, 2019), por esto se considera la aplicación de un sistema de bioseguridad en la zona de estudio para la prevención y atención a personas vulnerables ante la evolución y propagación de la enfermedad, cumpliendo así uno de los objetivos del Plan Estratégico Institucional en cuanto al cuidado de la salud e integridad de los ciudadanos.

La importancia de renovar el sector que forma parte de la vía férrea, siendo un patrimonio cultural de la urbe toma referencia en el Objetivo de Desarrollo Sostenible $N^{\circ} 3$ de Salud y Bienestar (ODS, 2016), donde la meta $\mathrm{N}^{\mathrm{a}} 3.9$ indica "Para el 2030, reducir sustancialmente el número de muertes y enfermedades producidas por productos químicos peligrosos y la contaminación del aire, el agua y el suelo", ya que se necesita de una intervención que brinde un control en la disminución de los residuos puesto que el personal de la alcaldía solicita a los moradores no arrojar desperdicios mientras realizan la limpieza en mingas comunitarias de salubridad.

\section{Sistema de sostenibilidad y reactivación económica}

El Sistema de sostenibilidad ambiental manifiesta como objetivo la recuperación urbana de zonas verdes para el uso recreativo y turístico, además de la rehabilitación de los espacios públicos (PEI Durán, 2019), siendo el sitio de estudio el indicado ya que se necesita el mejoramiento de sus condiciones urbanas, así mismo el [ODS] de Ciudades y comunidades sostenibles con meta $\mathrm{N}^{\mathrm{o}} 11.7$ indica que "De aquí a 2030, proporcionar el acceso a zonas verdes y espacios públicos seguros, inclusivos y accesibles, en particular para las mujeres, los niños, personas de edad y las personas con discapacidad".

La integración económica es uno de los aspectos que se plantea en base a los objetivos del sistema de desarrollo productivo del cantón y el plan nacional de desarrollo toda una vida, de manera que se promueva el manejo sostenible y el respeto a la naturaleza para un desarrollo económico con Buen Vivir, ya que el [ODS] de Trabajo decente y crecimiento económico, cuya meta 8.a ampara a "Aumentar el apoyo a la iniciativa de ayuda para el comercio en los países en desarrollo, en particular los países menos 
adelantados, incluso mediante el Marco Integrado Mejorado para la Asistencia Técnica a los países menos adelantados en Materia de Comercio".

Debido a esto, la revitalización del sitio es una alternativa ideal de repensar la extensión de 1'153.06 m existente en la línea férrea para el uso público, de manera que responda a las necesidades de los habitantes en erradicar los problemas sociales como la inseguridad debido al vandalismo y ambientales por la contaminación generada como los vertederos de basura que produce efectos perjudiciales para la salud pública, teniendo en cuenta la participación ciudadana; "debido a que un área verde segura y bien mantenida proporciona beneficios múltiples a la comunidad que rodea y para que ésta permanezca viable resulta no solamente de la provisión sostenible de fondos sino de la participación ciudadana" (Sorensen Mark, 1998). Con el objetivo de proponer la revitalización al área pública con la aplicación de criterios de diseño urbanístico ambiental y movilidad urbana para el mejoramiento de la calidad de vida de los habitantes del sector 5ta etapa y 3era etapa de la ciudadela el Recreo, del cual se derivan los objetivos específicos orientados a la investigación y propuesta planteada.

La importancia de fomentar una propuesta que revitalice el trayecto espacial abandonado proporciona una orientación en el pensamiento de los ciudadanos y las autoridades públicas a una cultura ambiental que actualmente se necesita en la zona de la ciudadela El Recreo, "se pretende beneficiar a los habitantes de estas zonas, mediante la implementación de áreas verdes y servicios, que les provean de espacios para un permanente contacto con la naturaleza y su entorno y permita estructurar ciudades con hábitat sustentables que brinden armonía entre el ambiente natural y el espacio construido que contribuya a alcanzar el Buen Vivir” (MIDUVI, 2015) ; además de solventar las variables en cuanto a la estética y funcionalidad del área junto a la línea férrea. En particular, la propuesta se enfoca en el planteamiento de un diseño ecológico y característico en el cual se incorporan elementos de estructuración en la vía pública y de confort urbano como los mobiliarios, pérgolas, quioscos, bolardos, pavimentación, entre otros, con el propósito de recuperar el espacio público y se mantengan las actividades de recreación para los habitantes del sector.

\section{ESTRATEGIAS METODOLÓGICAS O MATERIALES Y MÉTODOS}

Para el diagnóstico de la parroquia El Recreo se aplicó la investigación cuantitativa y cualitativa, ya que se requiere obtener una recopilación de datos numéricos por medio de 
las encuestas, pero también se requiere conocer el significado y la observación de la percepción de un problema desde el punto de vista de la persona que lo está viviendo, generando una respuesta real de tema a cuestionar. (Cervantinos, 2020)

Para conocer las cualidades que requieran las personas adquirir dentro de un espacio público se deberá conocer cuáles son las características de las que carece el sector y cuáles son las que posee actualmente, logrando identificar los pro y los contra del espacio a diseñar, por lo cual una vez ya obtenido esta información recurrimos a la metodología del arquitecto y urbanista (Ghel, 2017), nos indica que para crear espacios para la gente se deberán considerar cinco ámbitos de actuación, los cuales son: preservación del patrimonio, movilidad sustentable, equidad y diversidad, diseño urbano a escala humana, fomento económico y cultural.

Una vez ya identificado los ámbitos de Jan Ghel se procedió a la conceptualización de lo que se observó en cada ámbito, comenzando por el de preservación del patrimonio, el cual nos indica, que se deberá proteger y defender las especies arbóreas que se encuentren dentro del espacio de estudio, ya que cumplen con funciones en cuanto a la ecología del terreno, y la vida de muchas especies de animales. Otro dato a proteger es el valor histórico que se encuentre dentro de un objeto o espacio determinado, dándole valor e importancia al transporte ferroviario que se encuentra atravesando de una forma simétrica el terreno total de estudio. (Lopera, 2005)

Otro ámbito a tratar por el urbanista es la movilidad sustentable en el que se tratar de evitar la progresiva invasión de los vehículos, los cuales son el principal medio de transporte que genera mayor contaminación al medio ambiente y a los residentes que se encuentra a orillas de las vías vehiculares, por lo tanto se deberá optar por soluciones más sustentables y dar prioridad a la circulación peatonal, siendo este nuestro objetivo a alcanzar dentro de las vías que den acceso a la propuesta de un parque lineal dentro del terreno analizado. (Carmen, 2006)

La equidad y diversidad es otro de los aspectos a considerar para evitar que dentro del proyecto a proponer no se generen ningún tipo de segregación a grupos o individuos que no formen parte de los residentes del sector analizado, ya que una de las características principales de este ámbito es la equidad y diversidad dentro de un espacio público promoviendo el libre acceso para todos, generando oportunidades de conocernos entre individuos con diferentes características físicas y culturales, siendo así uno de los 
espacios que promueva la superación de los perjuicios y brinden una conexión emotiva entre los residentes y los visitantes. (Tovar, 2014)

La escala Humana es uno de los aspectos visuales y funcionales más importante para el ser humano, ya que a través de la historia se ha ido perdiendo el valor estético y emocional que provocaban los espacios que eran diseñados para el ser humano. Por lo tanto, el mensaje que propone (Ghel, 2017) en este aspecto, es el de implementar diseños de espacios que no solo cumplan con las necesidades y la antropometría del ser humano, sino que también mejore la experiencia visual con respecto a la arquitectura y genere emociones a través del recorrido de los que se percibe por medio de la vista.

En el ámbito del aspecto económico y cultural, indica que se deberá fomentar una relación entre la vida pública y el desarrollo económico, siendo esta una de las formas más eficaces de crear espacios sociables, fortaleciendo la actividad comercial y atrayendo nuevas inversiones dentro del espacio público a diseñar, por cual dentro del diseño del proyecto se idealiza crear un corredor comercial o islas comerciales a través del tramo lineal donde circularan los peatones, con el fin de lograr una reactivación económica y cultural del sector que actualmente se encuentra en un estado de abandono. (Jordán, Riffo, \& Prado, 2017)

\section{Criterios para el diagnóstico del espacio público de calidad}

Para lograr una buena justificación de las intervenciones en el espacio público en cuanto al diseño, se optó por realizar un registro de la vida pública siendo este subdividido en dos tipos de mediciones, que son: Conteo por edad y género, conteo de personas en movimiento. En el cual se realizó por las visitas de campo, y mediante la visualización se registraron los datos de acuerdo a la hora y día estimado, siendo estos establecidos en los dos métodos para generar un solo conjunto de respuestas obteniendo un valor más exacto del conteo de personas e identificando sus necesidades en cuanto a la circulación. (Rojas, Gallardo, \& Piñero, 2020) 


\section{Tabla 1}

Conteo por edad y género

\begin{tabular}{|c|c|c|c|c|c|c|c|c|c|c|}
\hline \multicolumn{2}{|c|}{ Dias } & \multicolumn{3}{|c|}{ Lunes } & \multicolumn{3}{|c|}{ Miércoles } & \multicolumn{3}{|c|}{ Viernes } \\
\hline Género & $\begin{array}{l}\text { Edad - } \\
\text { Años }\end{array}$ & $\begin{array}{c}9: 00- \\
9: 30 \mathrm{am}\end{array}$ & $\begin{array}{c}13: 00- \\
13: 30 \\
\text { pm }\end{array}$ & $\begin{array}{c}19: 00- \\
19: 30 \\
\mathrm{pm}\end{array}$ & $\begin{array}{c}9: 00- \\
9: 30 \mathrm{am}\end{array}$ & $\begin{array}{c}13: 00- \\
13: 30 \\
p m\end{array}$ & $\begin{array}{c}19: 00- \\
19: 30 \\
\mathrm{pm}\end{array}$ & $\begin{array}{c}9: 00- \\
9: 30 \mathrm{am}\end{array}$ & $\begin{array}{c}13: 00- \\
13: 30 \\
\mathrm{pm}\end{array}$ & $\begin{array}{c}19: 00- \\
19: 30 \\
p m\end{array}$ \\
\hline \multirow{6}{*}{ 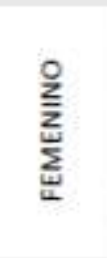 } & $0-10$ & 1 & 0 & 1 & 0 & 1 & 1 & 1 & 0 & 1 \\
\hline & oct-20 & 1 & 0 & 3 & 3 & 0 & 2 & 1 & 2 & 3 \\
\hline & $20-30$ & 2 & 1 & 3 & 2 & 2 & 2 & 2 & 1 & 3 \\
\hline & $30-40$ & 1 & 2 & 2 & 1 & 1 & 1 & 1 & 1 & 2 \\
\hline & $40-50$ & 3 & 2 & 1 & 1 & 2 & 2 & 2 & 1 & 1 \\
\hline & $50+$ & 1 & 0 & 0 & 1 & 1 & 1 & 0 & 1 & 1 \\
\hline \multirow{6}{*}{ 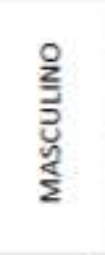 } & $0-10$ & 2 & 1 & 1 & 1 & 0 & 1 & 1 & 1 & 2 \\
\hline & oct-20 & 1 & 1 & 2 & 1 & 0 & 4 & 2 & 2 & 3 \\
\hline & $20-30$ & 1 & 1 & 2 & 1 & 1 & 1 & 1 & 1 & 3 \\
\hline & $30-40$ & 1 & 1 & 3 & 2 & 2 & 2 & 1 & 1 & 1 \\
\hline & $40-50$ & 1 & 1 & 1 & 0 & 1 & 1 & 1 & 2 & 1 \\
\hline & $50+$ & 0 & 0 & 1 & 1 & 1 & 0 & 0 & 1 & 1 \\
\hline \multicolumn{2}{|c|}{ Total } & 15 & 10 & 20 & 14 & 12 & 18 & 13 & 14 & 22 \\
\hline \multicolumn{2}{|c|}{ Total por dias } & \multicolumn{9}{|c|}{138} \\
\hline
\end{tabular}

Fuente: Elaboración propia

Tabla 2

Conteo por modo de desplazamiento

\begin{tabular}{|c|c|c|c|c|c|c|c|c|c|c|}
\hline \multirow{2}{*}{$\begin{array}{c}\text { Dias } \\
\text { Actividades/ } \\
\text { Horas }\end{array}$} & \multicolumn{3}{|c|}{ Lunes } & \multicolumn{3}{|c|}{ Miércoles } & \multicolumn{3}{|c|}{ Viernes } & \multirow[b]{2}{*}{$\begin{array}{l}\text { Total por } \\
\text { actividad }\end{array}$} \\
\hline & $\begin{array}{l}9.00- \\
9: 30 \\
9 m\end{array}$ & $\begin{array}{c}13.00- \\
13.30 \mathrm{pm}\end{array}$ & $\begin{array}{c}19.00- \\
19.30 \\
\text { pm }\end{array}$ & $\begin{array}{c}9.00-9.30 \\
\mathrm{~m}\end{array}$ & $\begin{array}{l}13: 00- \\
13: 30 \mathrm{pm}\end{array}$ & $\begin{array}{c}19.00- \\
19.30 \\
\mathrm{pm}\end{array}$ & $\begin{array}{c}9: 00-9: 30 \\
\text { am }\end{array}$ & $\begin{array}{l}13.00- \\
1330 \mathrm{pm}\end{array}$ & $\begin{array}{c}19.00- \\
19 \cdot 30 \\
\text { pm }\end{array}$ & \\
\hline $\begin{array}{c}\text { Estado de } \\
\text { Ocio }\end{array}$ & 2 & 0 & 3 & 1 & 1 & 3 & 1 & 1 & 3 & 15 \\
\hline $\begin{array}{l}\text { Persomas en } \\
\text { silla de } \\
\text { ruedas }\end{array}$ & 0 & 1 & 0 & 0. & 1 & 0 & 0 & 1 & 0 & 3 \\
\hline Peatones & 9 & 7 & 12 & 10 & 7 & 10 & 7 & 9 & 10 & 81 \\
\hline Cicliutas & 4 & 2 & 5 & 3 & 3 & 5 & 5 & 3 & 9 & 39 \\
\hline $\begin{array}{l}\text { Vehiculo } \\
\text { privado }\end{array}$ & 5 & 2 & 8 & 4 & 3 & 6 & 3 & 2 & 7 & 40 \\
\hline $\begin{array}{l}\text { Transporte } \\
\text { públicoilbus) }\end{array}$ & 7 & E & 7 & 8 & 6 & 9 & 7 & 9 & 7 & 65 \\
\hline Total & 27 & 20 & 35 & 26 & $\mathbf{2 1}$ & 33 & 23 & 25 & 36 & 246 \\
\hline
\end{tabular}

Fuente: Elaboración propia 


\section{Fórmula 1}

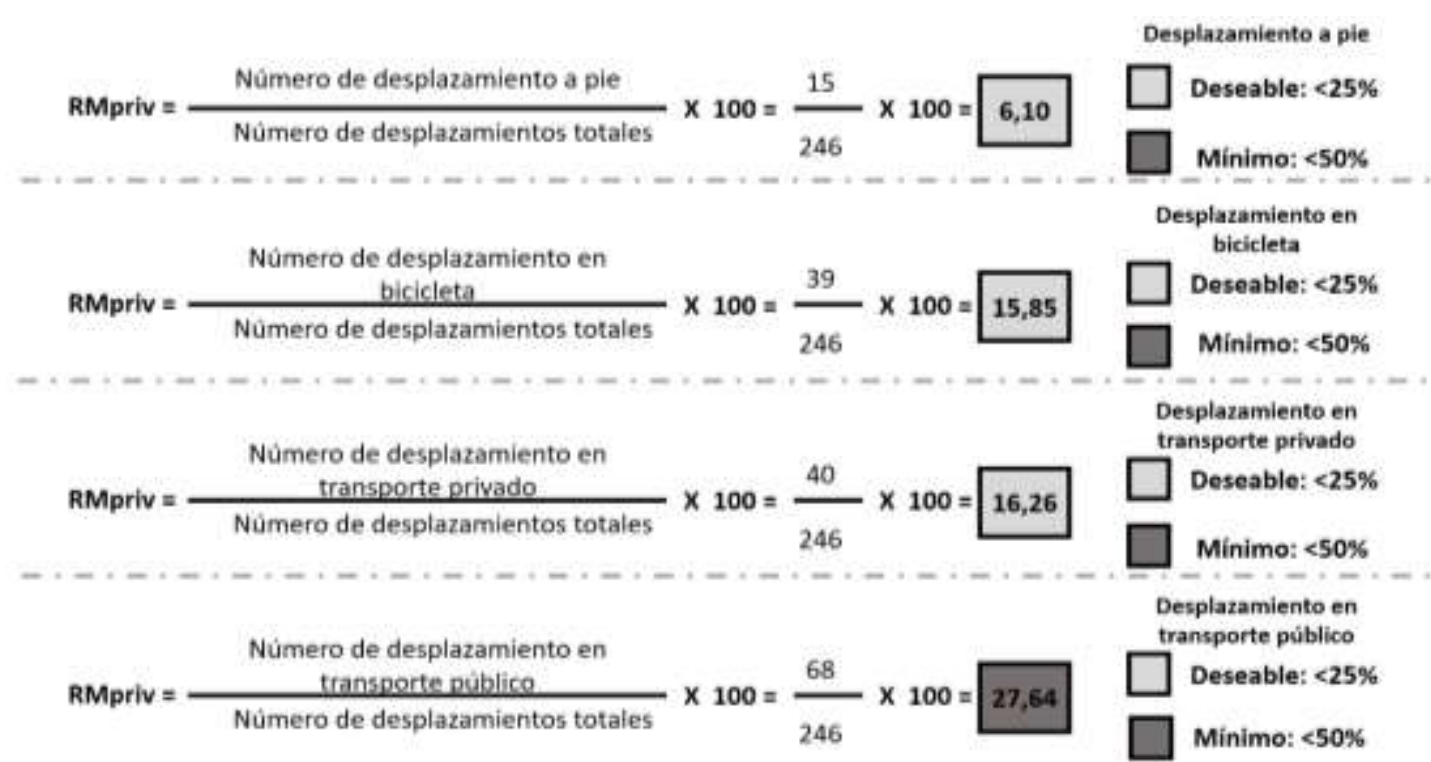

Una vez analizado y establecidos los aspectos principales en cuanto a el urbanismo de la metodología de Jan Ghel, se procederá a definir los criterios que se van a considerar para la definición del proyecto. Optando por la calificación de los criterios estimados según su grado de importancia, clasificándolos por tablas y temas generales que facilitaran el uso correcto del criterio a aplicar en cada área de estudio, siendo la letra A como el criterio más primordial, la letra $\mathrm{B}$ como el criterio considerable y la letra $\mathrm{C}$ como el criterio que no se va considerar dentro del diseño.

\section{Imagen 1}

\section{Criterios de calidad - Protección}

\begin{tabular}{|c|c|c|c|c|c|c|}
\hline & & Evaluador 1 & Evaluador 2 & Total & $\%$ & Condición \\
\hline & Protección del tráfico motorizado & & & & & $\mathrm{C}$ \\
\hline$z$ & Protección para peatones y ciclistas & 0,25 & 0,25 & 0,25 & $25 \%$ & \\
\hline$\frac{u}{u}$ & Acceso directo y seguro & 0 & 0 & 0 & $0 \%$ & $16 \%$ \\
\hline 岁 & Cruces seguros & 0 & 0 & 0 & $0 \%$ & 107 \\
\hline o & Tráfico de baja velocidad & 0,5 & 0,25 & 0,375 & $38 \%$ & \\
\hline$\stackrel{\leftrightarrow}{a}$ & $\begin{array}{l}\text { Protección del crimen y la violencia } \\
\end{array}$ & & & & & $\mathrm{C}$ \\
\hline '́ & Espacio urbano activo & 0,25 & 0,75 & 0,5 & $50 \%$ & \\
\hline 远 & Vigilancia pasiva & 0,25 & 0 & 0,125 & $13 \%$ & $19 \%$ \\
\hline$\overline{\bar{u}}$ & Diversidad de funciones & 0 & 0 & 0 & $0 \%$ & \\
\hline $\begin{array}{c}u \\
w\end{array}$ & Iluminación adecuada y atractiva & 0 & 0,25 & 0,125 & $13 \%$ & \\
\hline 㟧 & Protección de experiencias sensoriales desagradables & & & & & $\mathrm{C}$ \\
\hline$\frac{\tilde{o}}{\tilde{u}}$ & $\begin{array}{l}\text { Protección del sol, lluvia, vientos y otros elementos } \\
\text { climáticos }\end{array}$ & 0,25 & 0,25 & 0,25 & $25 \%$ & \\
\hline$\frac{E}{\alpha}$ & Protección del frío o el calor & 0 & 0 & 0 & $0 \%$ & $9 \%$ \\
\hline 崩 & Bajos niveles de ruido & 0 & 0,25 & 0,125 & $13 \%$ & \\
\hline & Bajo nivel de contaminación o aromas desagradables & 0 & 0 & 0 & $0 \%$ & \\
\hline
\end{tabular}




\section{Imagen 2}

\section{Criterios de calidad - Confort}

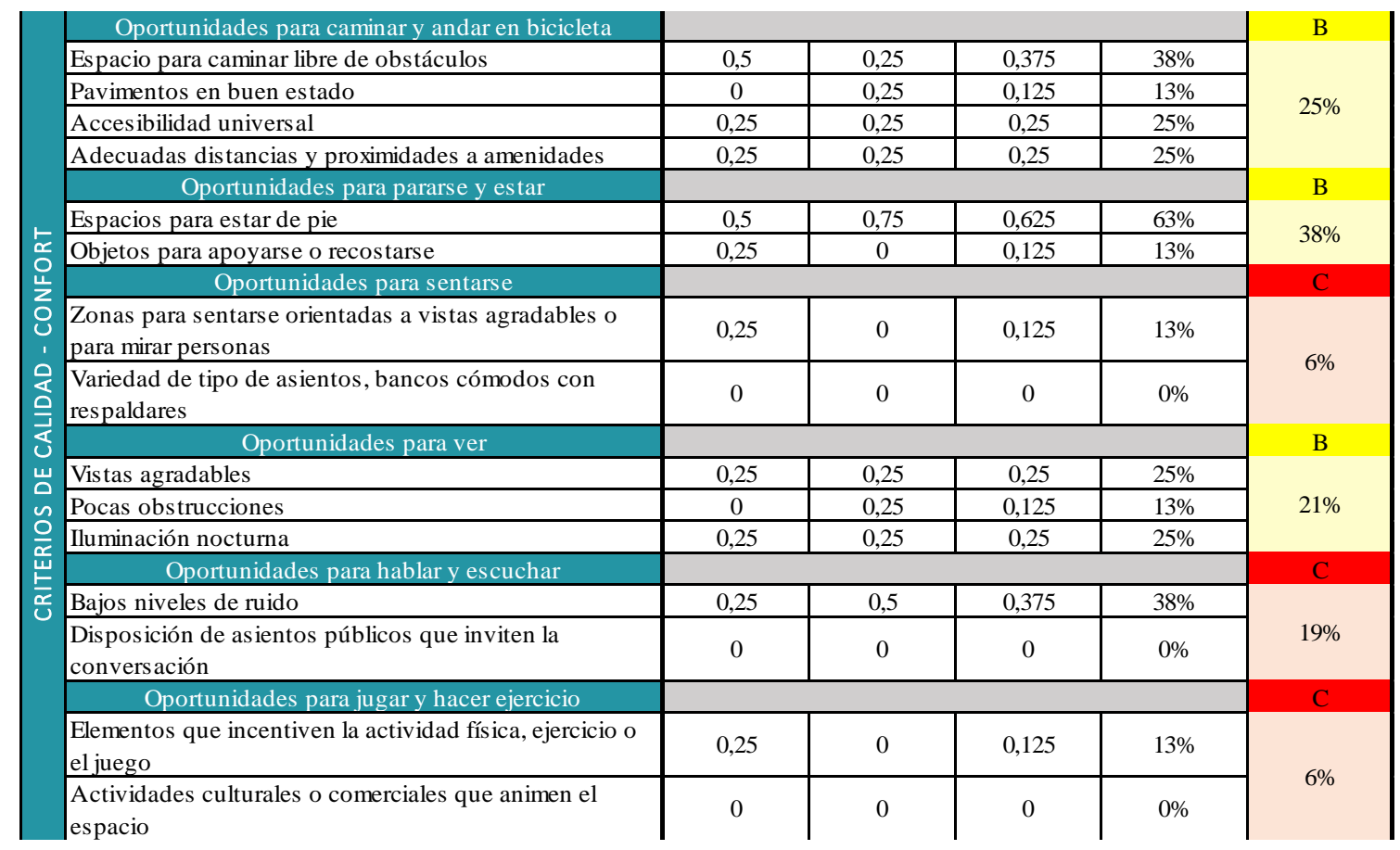

\section{Imagen 3}

\section{Criterios de calidad - Placer}

\begin{tabular}{|c|c|c|c|c|c|c|}
\hline \multirow{3}{*}{ 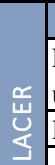 } & Dimensionado a la escala humana & & & & & $\mathrm{B}$ \\
\hline & $\begin{array}{l}\text { Espacio con dimensiones apropiadas para su nivel de } \\
\text { uso cotidiano }\end{array}$ & 0,25 & 0,5 & 0,375 & $38 \%$ & \multirow{3}{*}{$25 \%$} \\
\hline & Distancias apropiadas entre los elementos & 0,25 & 0,5 & 0,375 & $38 \%$ & \\
\hline \multirow{3}{*}{ 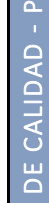 } & $\begin{array}{l}\text { Mobiliario y elementos arquitectónicos proporcionados } \\
\text { a escalas adecuadas y cómodas para el cuerpo humano }\end{array}$ & 0 & 0 & 0 & $0 \%$ & \\
\hline & Oportunidades para disfrutar del clima y la naturaleza & & & & & $\mathrm{B}$ \\
\hline & $\begin{array}{l}\text { Orientación que permita aprovechar el sol, la sombra, } \\
\text { brisas, entre otros }\end{array}$ & 0 & 0,5 & 0,25 & $25 \%$ & \multirow[t]{2}{*}{$38 \%$} \\
\hline \multirow{5}{*}{$\begin{array}{l}\frac{\sim}{0} \\
\frac{\mathrm{O}}{\mathrm{w}} \\
\frac{\mathrm{E}}{\mathrm{c}}\end{array}$} & Árboles y vegetación apropiada para la región & 0,5 & 0,5 & 0,5 & $50 \%$ & \\
\hline & Cualidades estéticas + experiencias sensoriales positivas & & & & & $\mathrm{C}$ \\
\hline & Buen diseño, materiales y detalles & 0 & 0 & 0 & $0 \%$ & \multirow{3}{*}{$13 \%$} \\
\hline & Buenas vistas, orientación apropiada & 0 & 0,25 & 0,125 & $13 \%$ & \\
\hline & Experiencias sensoriales agradables, naturaleza, plantas & 0,25 & 0,25 & 0,25 & $25 \%$ & \\
\hline \multirow{3}{*}{\multicolumn{2}{|c|}{$\begin{array}{l}\text { Observación: Los criterios que cumplan entre el } 50 \% \text { al } 75 \% \text { como } \\
\text { factor mínimo deseable, indican que el espacio público evaluado es } \\
\text { adecuado para la vida pública. }\end{array}$}} & $\mathbf{A}$ & $>75 \%$ & \multicolumn{3}{|c|}{ Cumple con todos los criterios } \\
\hline & & B & $25 \%-75 \%$ & \multicolumn{3}{|c|}{ Cumple con los criterios a medias } \\
\hline & & $\mathbf{C}$ & $<25 \%$ & \multicolumn{3}{|c|}{ No cumple con todos los criterios } \\
\hline
\end{tabular}




\section{RESULTADOS Y DISCUSIÓN}

El espacio público a tratar dentro de la parroquia el recreo muestra a través del análisis realizado, que el pueblo requiere de una revitalización del espacio público que actualmente se encuentra en estado de abandono, por lo cual muestra como resultado la implementación de distintos tipos de propuestas con el fin del resolver las necesidades presentadas por el diario vivir de los moradores del sector. Estableciendo una tabla (ver tabla 3) con los resultados de acuerdo a los distintos tipos de desplazamiento, mostrando el valor del diagnóstico actual, el valor estándar y el valor incrementado según la propuesta por cada desplazamiento.

\section{Tabla 3}

Comparativa entre lo actual y la propuesta

\begin{tabular}{|cccc|}
\hline $\begin{array}{c}\text { Desplazamiento a } \\
\text { pie }\end{array}$ & Criterio ideal & Diagnostico & Propuesta \\
\hline $\begin{array}{c}\text { Transporte a pie } \\
\begin{array}{c}\text { Desplazamiento en } \\
\text { bicicleta } \\
\text { Transporte en } \\
\text { bicicleta }\end{array}\end{array}$ & $25 \%$ & $6,10 \%$ & $40 \%$ \\
$\begin{array}{c}\text { Desplazamiento en } \\
\text { transporte privado }\end{array}$ & Criterio ideal & Diagnostico & Propuesta \\
$\begin{array}{c}\text { Transporte privado } \\
\text { Desplazamiento en } \\
\text { transporte público }\end{array}$ & $25 \%$ & $15,85 \%$ & $40 \%$ \\
\hline \begin{tabular}{c} 
Transporte público \\
\hline
\end{tabular} & Criterio ideal & $16,26 \%$ & $10 \%$ \\
\hline
\end{tabular}

\section{Fuente: Elaboración propia}

Uno de los principales factores por el cual el espacio se encontraba en muy malas condiciones fueron los vertederos de basura ya que, dentro del espacio a tratar, no se encuentra ningún tipo de depósito de basura adecuado, por tal motivo se empleó implementar cada 50 metros un contenedor, según los resultados mostrados por la formula aplicada. 


\section{Fórmula 2}

Fórmula: dotación de contenedores

$$
\mathrm{DC}=\frac{\text { Población total }}{\text { Números contenedores }}=\frac{378}{20}=18,9
$$

Fórmula: distancia acceso

$$
\mathrm{DC}=\frac{\text { Distancia de acceso }}{\text { Números contenedores }}=\frac{50}{20}=2,5
$$

\section{Cantidad de contenedores}

Deseable: $100 \mathrm{~m}$

habitantes/contenedor

Minimo : $300 m$

habitante/contenedor

\section{Acceso a contenedores}

Deseable $50 \mathrm{~m}$

Minimo $100 \mathrm{~m}$

\section{Imagen 4}

\section{Ubicación de contenedores según la fórmula}

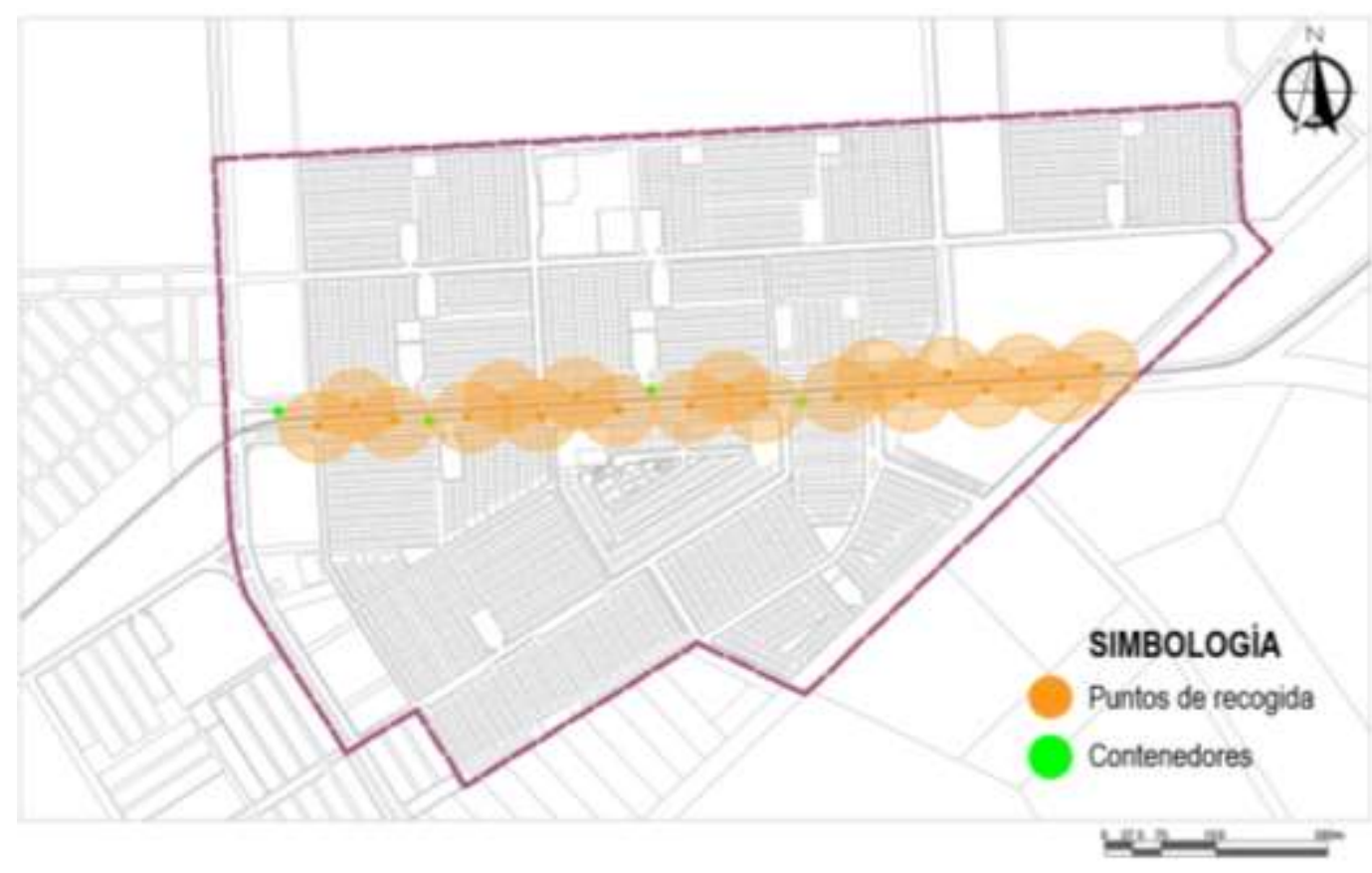

Siendo otro de los factores por el cual el sitio se suele encontrar inaccesible, es el estancamiento de aguas lluvias en los tiempos de invierno, por lo cual se realizó una identificación mediante la fórmula de permeabilidad (ver formula 3), obteniendo como resultado un valor real de la cantidad de suelo permeable actual sobre el valor de la cantidad de suelo permeable obtenido mediante la revitalización. 


\section{Fórmula 3}

ACTUAL

$$
\text { Ap }=\frac{\text { Metros permeables }}{\text { Metros totales de intervención }}=\frac{1.952}{79.623,55}=0,02 \mathrm{m2}
$$

PROPUESTA

$$
A p=\frac{\text { Metros permeables }}{\text { Metros totales de intervención }}=\frac{32.763,1}{79.623,55}=0,41 \mathrm{m2}
$$

\section{Fórmula 4}

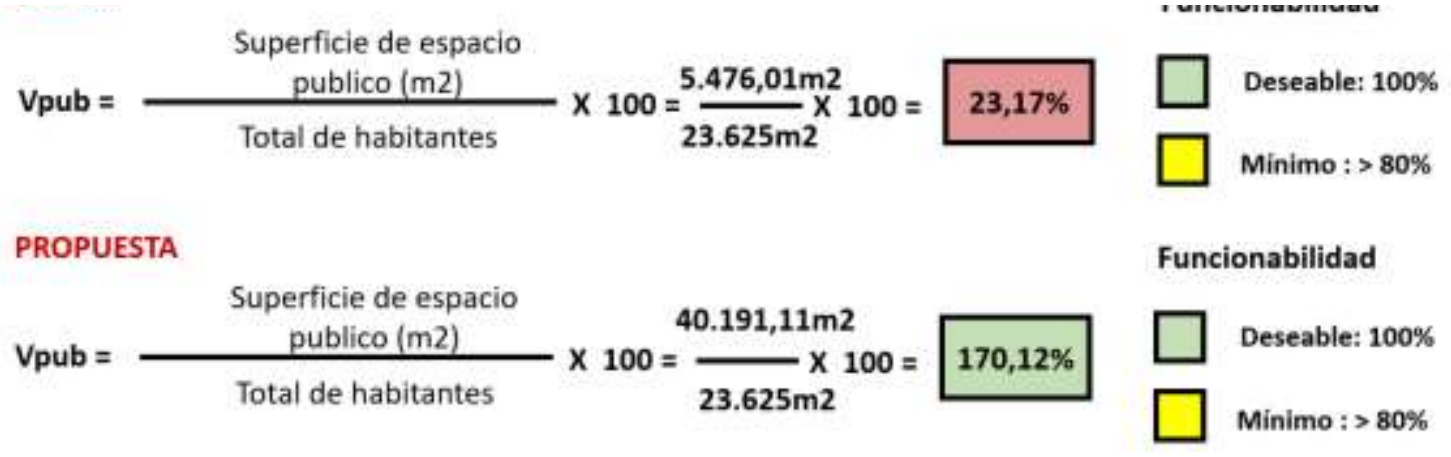

\section{Imagen 5}

\section{Estado actual del terreno}

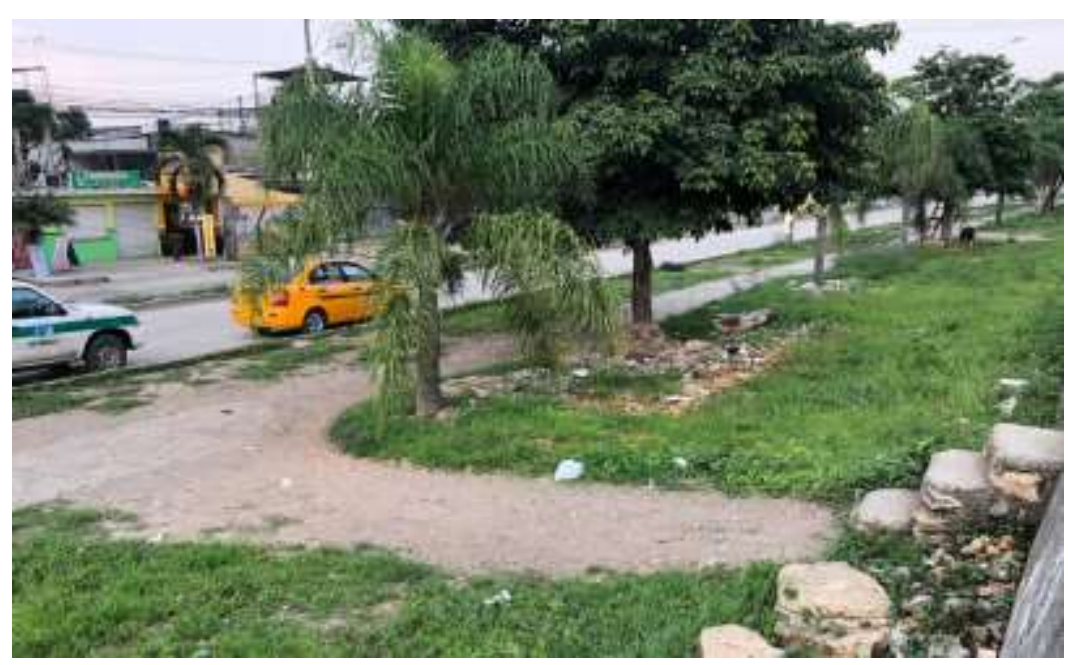




\section{Imagen 6}

\section{Terreno con la implementación del proyecto}

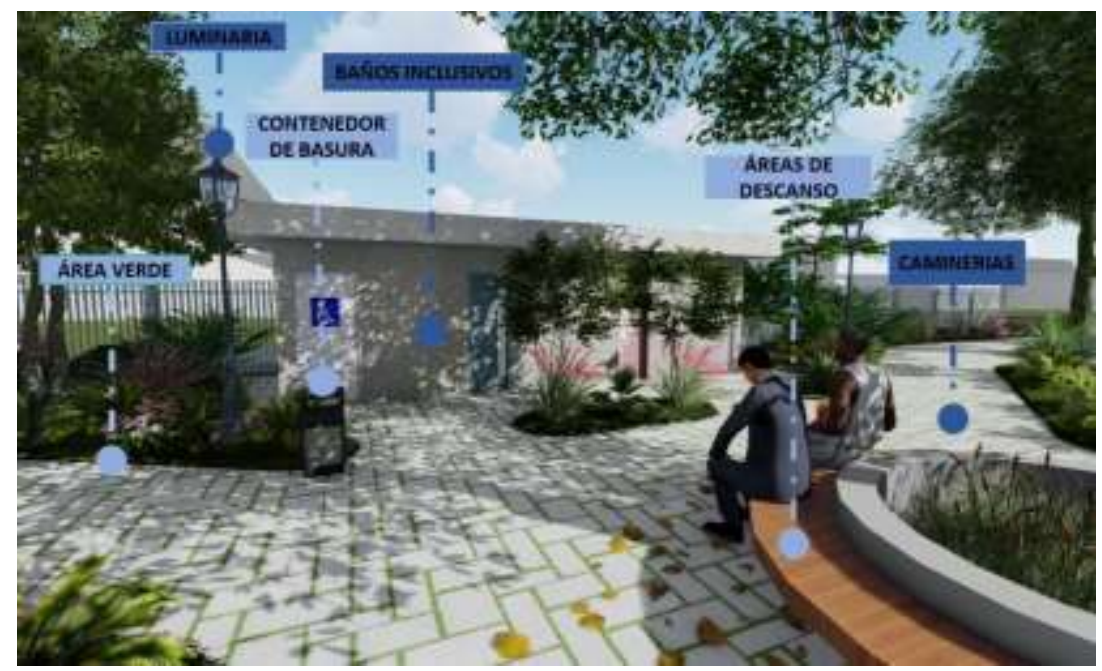

Luego de haber intervenido en muchos aspectos dentro de un espacio público en estado de abandono, se puede evidenciar que la funcionabilidad de los espacios públicos era tan insignificante para una gran cantidad de personas, que en su diario vivir buscaban un espacio en común con presencia de zonas verdes puesto que estas con mejores niveles de salud mental en la población, al actuar como reguladores de estrés, ansiedad y depresión. Por lo cual, al realizar una revitalización de un espacio conector, este espacio funcional aumenta en un grado tan significativo (ver formula 4), que sobrepasa el valor del porcentaje deseable. (Merayo, 2016)

\section{CONCLUSIÓN O CONSIDERACIONES FINALES}

Ante el estado de desinterés de las autoridades del cantón por proponer un parque lineal en un espacio inutilizado, los moradores del sector se han visto con la necesidad de transitar por el espacio abandonado por medio de vehículos, o circular en ciertas horas del día, ya que al encontrarse con aceras inaccesibles o con una falta de iluminación que a su vez provoca la inseguridad, generando un alto grado de desinterés por volver a transitar por este sector, calificándolo por los visitantes como un lugar inseguro, trayendo consigo un déficit económico por partes de las tiendas ubicadas a orillas de las calles que rodean el espacio abandonado.

Pero que luego de haber calificado a este espacio como un lugar inseguro, en un tiempo donde aparece la llegada del virus COVID-19, trayendo consigo una paralización de las actividades; durando una gran cantidad de meses en estado de aislamiento, provoco una 
necesidad por parte de los habitantes de recrearse en un espacio abierto donde se mantenga una distancia deseable entre personas, por lo cual optaron por reutilizar este espacio abandonado, para realizar actividades físicas, actividades de recreación y ocio.

Olvidando la inseguridad y el mal estado del espacio, los moradores del sector fueron adecuando con restos de materiales de construcción, según sus necesidades e implementando horarios para que a ciertas horas del día se pueda salir en conjunto y evitar el sentimiento de inseguridad que genera el sitio; según el tramo del espacio optaron por depositar desechos en zonas donde no existe área verde, y en donde si existe área verde, lo tomaron como zonas de descanso. Mostrando con esto, que el espacio que se encuentra atravesado por el riel del tren, que a su vez es considerado patrimonio nacional, a través de la necesidad las personas fueron adaptándose a las irregularidades que presenta el sitio. Por este motivo, se concluyó en presentar una propuesta de revitalización en vista de las necesidades que presentaban los residentes del sector, con el fin de proponer una respuesta a los problemas que se fueron acumulando a través del tiempo, e identificando las soluciones y los beneficios que estas generaban en cada problema, evidenciando una mejora a la vista urbana de la parroquia El Recreo.

\section{LISTA DE REFERENCIAS}

Arrieta, A. (2017). Parque Lineal Ferrocarril de Cuernavaca. 2021, de plataforma arquitectura Sitio web: https://www.plataformaarquitectura.cl/cl/886566/parquelineal- ferrocarril-de-cuernavaca-gaeta-springall-arquitectos

GADMCD. (2019). Plan de Desarrollo y Ordenamiento Territorial. En PDOTGADMCD (118). Guayaquil: Departamento de Comunicación de la Consultoría.

ODS. (2016). ODS Salud y Bienestar. 2016, de Naciones Unidas Sitio web: https://www.un.org/sustainabledevelopment/es/health/

GAD Durán. (2019). Plan Estratégico Institucional Municipal. 2021, de GAD Durán Sitio web: http://www.duran.gob.ec/municipio/inicio/descarga/PEIDURAN.pdf Proyecto de Ley Orgánica De Ordenamiento Territorial, Uso y Gestión de Suelo. (2016) Sitio web: https://www.habitatyvivienda.gob.ec/wpcontent/uploads/downloads/2016/08/Ley-Organica-de-Ordenamiento-TerritorialUso-y-Gestion-de-Suelo1.pdf 
Cervantinos. (2020). 15 tipos de investigación científica. 2020, de Centro Estudios Cervantinos Sitio web: https://www.centroestudioscervantinos.es/tipos-deinvestigacion-y-caracteristicas/

Ghel, J. (2017). La dimensión humana en el espacio público. Chile: MINVU. Obtenido de: $\quad$ https://www.minvu.cl/wp-content/uploads/2019/08/La-dimensionhumana.pdf

Lizárraga C. Movilidad Urbana Sostenible: un reto para las ciudades del siglo XXI.

Economía, Sociedad y Territorio VI, núm. 22, 2006, 283-321 , vol. VI, núm. 22, 2006, 283-321. Sitio web: https://biblat.unam.mx/hevila/Economiasociedadyterritorio/2006/vol6/no22/1.pd $\underline{\mathrm{f}}$

Jordán R; Riffo L; Prado A. Desarrollo sostenible, urbanización y desigualdad en América Latina y el Caribe. Septiembre 2017. Página Digital de CEPAL. Sitio web:

https://repositorio.cepal.org/bitstream/handle/11362/42141/1/S1700701_es.pdf

Rojas, L., Gallardo, A., \& Piñero, A. (10 de febrero de 2020). Redisa. Obtenido de Redisa: http://www.redisa.net/doc/artSim2011/GestionYPoliticaAmbiental/La\%20distancia\%2d el\%20domicilio\%20al\%20contenedor\%20como\%20un\%20factor\%20influyente \%20en\%201a\%20frecuencia\%20de\%20separaci\%C3\%B3n\%20de\%20residuos \%20urbanos.pdf

Gómez Lopera F. Las zonas verdes como factor de calidad de vida en las ciudades. 2005. Fundación Española para la Ciencia y la Tecnología FECYT. Sitio web: https://recyt.fecyt.es/index.php/CyTET/article/view/75554/46036

Merayo Rodríguez J, Serrano Fuentes N, Marqués Sánchez P. Influencia de los espacios verdes urbanos en la salud mental. Metas Enferm nov 2016; 19(9): 20-26. Sitio web: https://www.enfermeria21.com/revistas/metas/articulo/80987/influenciade-los-espacios-verdes-urbanos-en-la-salud-mental/

Torres Tovar C. Equidad Urbana en el Desarrollo- Ciudades para la Vida. Bitácora Urbano Territorial vol.24 no.1 Bogotá Jan./June 2014. Sitio web: http://www.scielo.org.co/scielo.php?script=sci_arttext\&pid=S0124$\underline{79132014000100007}$ 
Ministerio de Desarrollo Urbano y Vivienda. Proyecto de inversión: generación de áreas verdes y espacios recreativos públicos para la Provincia del Guayas - Guayas ecológico. Enero 2015. Sitio web: https://www.habitatyvivienda.gob.ec/wpcontent/uploads/downloads/2016/02/Guayas-Ecologico_20_01_2016.pdf

Sorensen M; Barzetti V; Keipi K. Manejo de las áreas verdes urbanas. Washington, D.C. - No. ENV - 109. Mayo, 1998. Sitio web: https://edisciplinas.usp.br/pluginfile.php/80827/mod_resource/content/1/Manejo \%20de\%20las\%20areas\%20verdes\%20urbanas_BM_1997.pdf

Zarta P. La sustentabilidad o sostenibilidad: un concepto poderoso para la humanidad.

Tabula Rasa, núm. 28, pp. 409-423, 2018. Sitio web: https://www.redalyc.org/jatsRepo/396/39656104017/html/index.html 